\title{
The alpha defensin lateral flow test is effective in predicting eradication of periprosthetic joint infection after surgical debridement
}

\author{
Lucy C. Walker, Nick D. Clement, Munawar Hashmi, Stephen Green, Lee Longstaff, David J. Deehan
}

From the Foundation Trust, Freeman Hospital, Newcastle-upon-Tyne, UK

The primary aim of this study was to assess the utility of the alpha defensin lateral flow (ADLF) test for predicting the eradication of PJI after surgical debridement. The secondary aim was to describe the reliability of ADLF test in diagnosis of PJI intraoperatively. A prospective observational study was conducted in three independent orthopaedic centres. Twenty-two patients undergoing revision surgery (debridement, antibiotics and implant retention (DAIR), single or two-stage revision) for PJI were recruited, 13 female and 9 male with an average age of 64 years. Samples were collected intra-operatively at the start of the first surgical procedure and then at the completion of debridement or prior to reimplantation depending on the operation performed. These samples were tested using ADLF and then sent for microbiological analysis. The ADLF result was then compared to the corresponding culture result in order to determine the diagnostic predictive accuracy. The reliability of ADLF test to predict eradication of infection after debridement of PJI was excellent for specificity and positive predictive value (PPV) of which both where $100 \%$, but had a poor sensitivity $(14.3 \%)$ and negative predictive value (NPV) $(62.5 \%)$. The reliability of ADLF test to predict PJI was poor with only a $50 \%$ sensitivity and specificity. The ADLF test has a high specificity and PPV for diagnosing eradication of infection after debridement. In contrast the ADLF testing appears to have poor diagnostic accuracy for PJI when used on intra-operative samples, prior to surgical intervention.

No benefits or funds were received in support of this study. None of the authors have a conflict of interest.
Keywords : Periprosthetic joint infection ; alpha defensin lateral flow test ; revision surgery ; infection eradication.

\section{INTRODUCTION}

Periprosthetic joint infection (PJI) is the second most common complication of joint arthroplasty (1). Early diagnosis of an acute infection may be treated with thorough irrigation and debridement of the infected prosthesis followed by tailored antibiotic therapy (debridement, antibiotics and implant retention - DAIR procedure) with the original metal implants remaining in situ thereby potentially

- Lucy C Walker ${ }^{1}$, MSc MPhil MRCS

- Nick D. Clement ${ }^{1}$, PhD FRCS

- Munawar Hashmi ${ }^{1}$, MD FRCS

Stephen Green ${ }^{2}$, FRCS

- Lee Longstaff ${ }^{3}$, FRCS

- David J. Deehan ${ }^{1}$, DSc FRCS

${ }^{1}$ Newcastle-upon-Tyne NHS Foundation Trust, Freeman Hospital, Newcastle-upon-Tyne, UK.

${ }^{2}$ Sunderland City Hospitals NHS Foundation Trust, Sunderland Royal Hospital, Sunderland, UK.

${ }^{3}$ County Durham and Darlington NHS Foundation Trust, University Hospital of North Durham, Durham, UK.

Correspondence: Lucy C. Walker, Newcastle-upon-Tyne NHS Foundation Trust, Freeman Hospital, Freeman Road, Newcastle-upon-Tyne, NE7 7DN, UK.

Email : Lcwalker86@gmail.com

- 2021, Acta Orthopædica Belgica. 
avoiding the overt risks of bone destruction or loss, nerve or vessel injury and soft tissue compromise (2). It is known that infecting organisms can form a biofilm within the first two weeks of infective symptoms (3) and multiple authors argue that the presence of a biofilm mandates complete component exchange (2). Single stage, two in one revision arthroplasty has key proponents but should be reserved for cases where a sensitive organism has been identified (4). The gold standard remains twostage revision with temporary use of an antibioticloaded cement spacer and adjuvant antibiotic therapy (4). Re-implantation should be performed after confirmation of absence of residual infection. However, no single test is absolutely specific hence reliance of a number of serological markers over time.

It is agreed that the culture of aspirate or tissue biopsy offers the best chance of confirming absence of residual infection with no one single test performed being definitive $(5,6)$. Culture results are considered essential as they not only provide a diagnosis but also identify the specific responsible organisms which allows for sensitivity testing and a tailored antimicrobial treatment plan (4). However, the reliability of culture results is variable (7) and the time required to finalise culture results combined with their modest accuracy has prompted investigators to develop and define more rapid and reliable synovial fluid analyses for diagnosing PJI (8).

Alpha defensin is an antimicrobial peptide, which is released in the synovial fluid by polymorphonuclear cells in response to bacterial pathogens (9). A synovial fluid $\alpha$-defensin test has been specifically developed to aid the diagnosis of PJI (10) and an alpha defensin lateral flow (ADLF) test, Synovasure ${ }^{\circledR}$, has also been developed to allow for in-theatre results (11). However, its application in clinical use is limited by the fact that it does not identify a causative organism (12).

Whilst identification of the causative organism is invaluable at the time of initial diagnosis, the surgical decision making process is binary when it comes to timing of re-implantation. Hence use of a test or marker of infection which can be performed immediately could be invaluable if reliable and sensitive. When culture samples are taken prior to proceeding with a second stage revision reimplantation they have been shown to have poor sensitivity (13). The reported high rates of false negatives is felt to be due to persistent antibiotic elution from the cement spacer (13). However, alpha defensin levels have previously been shown not to be influenced by concurrent or recent antibiotic administration (14). The ADLF test can offer intraoperative results and can be performed during the pre-implantation / further debridement phase.

The primary aim of this study was to assess the utility of the ADLF test for predicting the eradication of PJI after surgical debridement. The null hypothesis was that ADLF test was a poor predictor of eradication of PJI after surgical debridement. The secondary aim was to describe the reliability of ADLF test in diagnosis of PJI intra-operatively.

\section{MATERIALS AND METHODS}

This was a prospective observational study investigating the use of the ADLF test in revision surgery for PJI of either hip or knee arthroplasty. Intra-articular synovial fluid samples taken at the start of the surgical procedure (DAIR, single or two stage revision), and at the completion of the chosen surgical intervention (at the end of debridement in DAIR procedures or prior to re-implantation if single or two stage revision) were compared to the chosen gold standard of the culture results from synovial fluid and tissue samples taken at the same time points. Patient recruitment was carried out within the orthopaedic department of three centres.

The cohort of patients included all patients undergoing surgical treatment for PJI of hip or knee arthroplasty. The diagnosis of PJI was defined as either a culture positive pre-operative joint aspiration or the clinical diagnosis made by the treating team if an aspiration had not been obtained or in instances of a 'dry' aspirate.

Figure 1 shows the experimental protocol pathway. All ADLF testing was performed under the supervision of the lead researcher as per the instruction leaflet published by ZimmerBiomet, the manufacturers of the product (15). For patients undergoing a DAIR or single stage revision surgery 


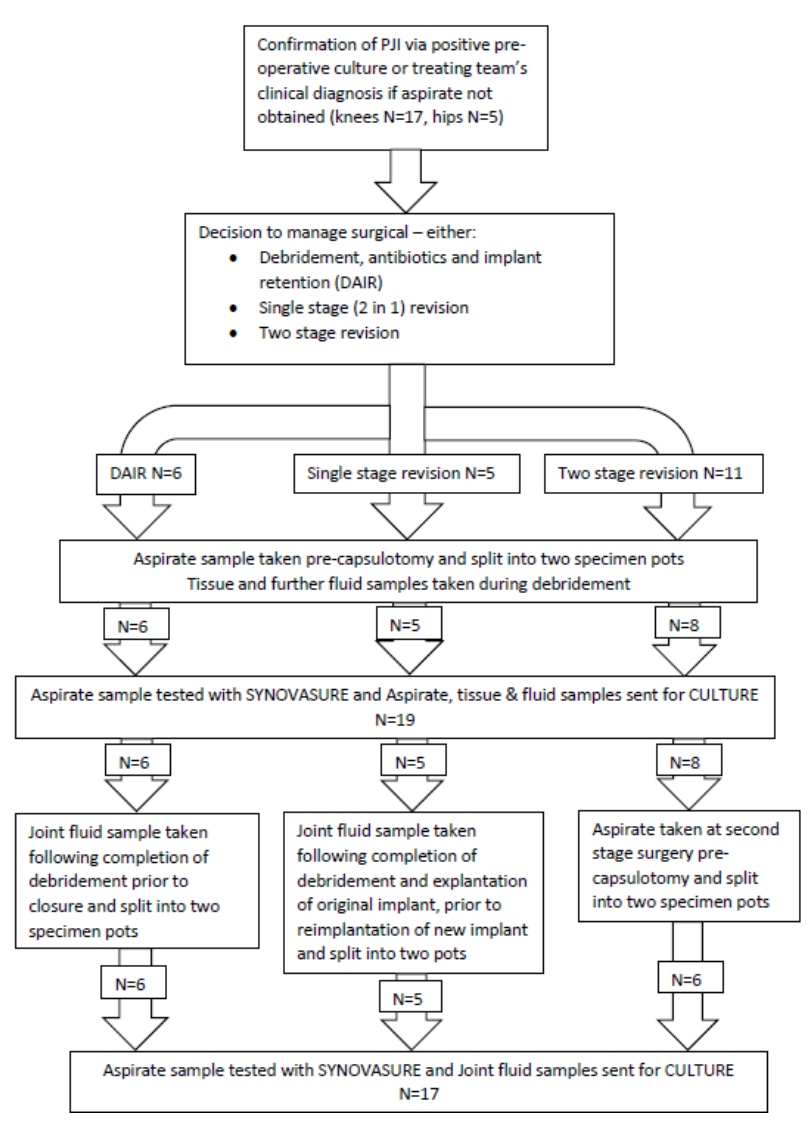

Fig. 1. - Flow diagram of experimental protocol

an aspirate of synovial joint fluid was taken prior to the capsulotomy. The joint aspirate was split by the scrub nurse, half was sent for culture and half was handed out, tested in theatre using an ADLF testing kit and the result recorded. Figure 2 shows the appearance of an ADLF test kit for both a positive and negative result. Multiple other tissue sample were then taken intra-operatively as per recommended practice (4) and sent for culturing. For a DAIR procedure a second joint fluid sample was taken after the operating surgeon was satisfied that the washout and debridement was complete. For a single stage revision the second fluid sample was taken once explantation, washout and debridement was complete, just prior to commencing with reimplantation. The second fluid sample was also split, half sent for culture, half tested in theatre with an ADLF test kit and the result recorded.

For patients undergoing a two stage revision surgery, the first synovial fluid sample was taken

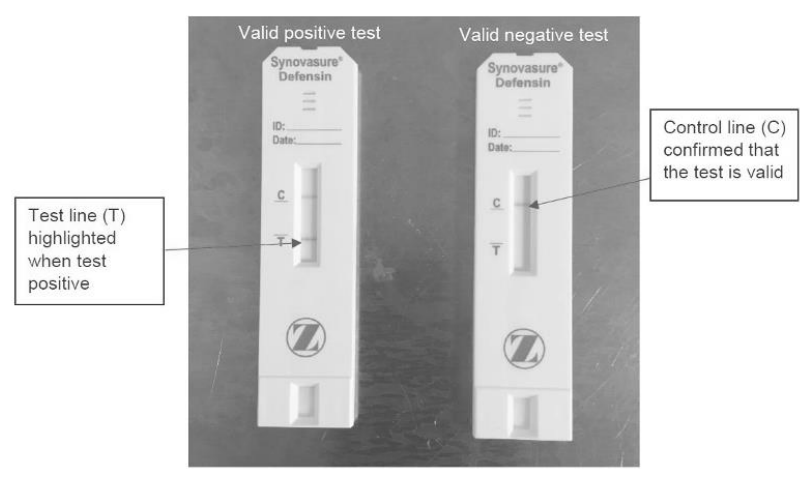

Fig. 2. - Synovasure test kits

at the start of the first stage revision surgery prior to the capsulotomy using the same technique as the DAIR and single stage cases. The second sample was then taken at the start of the second stage revision procedure, prior to the capsulotomy. Further intra-operative samples were also routinely taken and sent for culture during both the first and second stage surgeries.

Medical records, operation notes, haematological, microbiological and radiological results were also reviewed for each patient to collect data on patient demographics, medical and orthopaedic history, PJI presenting history, inflammatory markers including white cell count (WCC), C-reactive protein (CRP) and erythrocyte sedimentation rate (ESR), date of aspiration and final culture result, date and details of surgical management, antibiotic administration (pre, intra and post-operatively) and culture results of intra-operative samples. Patients with diabetes, peripheral vascular disease, rheumatological conditions, vascular disease, renal failure, hepatic failure and/or chronic pulmonary disease were defined as having a medical history 'at risk of PJI' (16).

The ADLF test results were then compared with the corresponding final culture results to determine the accuracy of the ADLF test kits at differentiating between a septic and aseptic joint both at the start and completion of revision surgery for PJI.

One way ANOVA was used to compare linear variables between operation groups. Dichotomous variables were assessed using a Chi square test. Simple descriptive statistics were undertaken to calculate the sensitivity, specificity, positive pre- 
dictive value and negative predictive value for predictors of PJI.

Health Research Authority (IRAS number 237090) and Research Ethics Committee approval (18/NE/0063) was obtained for the current study. Each patient provided written consent for their participation in the study, including the testing of synovial fluid samples and the review of their medical notes and test results. The study was registered with the lead centre's Research and Development department (registration number 08581).

\section{RESULTS}

The final cohort was compiled of 22 patients with a mean age of 64 years (range 30-92). 13 patients were female and 9 were male. Twelve $(54.5 \%)$ patients had a medical history that increased their risk of PJI. Seventeen (77.3\%) patients had revision surgery on a knee prosthesis and five had hip prostheses. Seven (31.8\%) patients were acute presentations of PJI, the remaining 15 patients had chronic PJI. The mean time between presentation and first surgery was 306 days (range 3-1116 days). The average time between the $1^{\text {st }}$ and $2^{\text {nd }}$ surgery was 52 days (range 0-273 days), this was calculated for 19 patients as 3 patients had a first stage revision surgery but did not proceed to a second stage surgery.

Six patients had a DAIR procedure, five had single stage (two in one) revision surgery and eleven

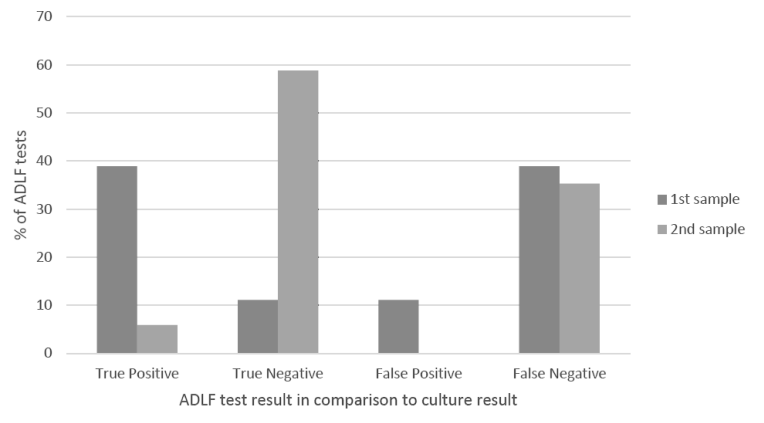

Fig. 3. - Comparison of ADLF test results and $1^{\text {st }}$ and $2^{\text {nd }}$ stages

patients underwent two stage revision surgery. Table 1 shows the patient demographics as per surgery type. There was no statistically significant difference between the three groups for age, sex, PMH or joint type. There was a statistically significant difference between the operation types for type of PJI (acute vs chronic), the time between presentation and $1^{\text {st }}$ surgery as well as time between the $1^{\text {st }}$ and $2^{\text {nd }}$ stages of surgery.

Figure 3 shows the ADLF results at the $1^{\text {st }}$ and $2^{\text {nd }}$ sampling in comparison to the gold standard of the comparable culture results. Table 2 shows the sensitivity, specificity, PPV and NPV for ADLF testing at the $1^{\text {st }}$ and $2^{\text {nd }}$ stages, using the corresponding sample culture results as the gold standard.

Five patients did not have ADLF or culture testing at the second sampling i.e. after debridement +/- explantation, so these patients were excluded from the subsequent analysis.

Table 1. - Demographics as per operation type

\begin{tabular}{|c|c|c|c|c|}
\hline & $\begin{array}{c}\text { DAIR } \\
\mathbf{N}=6\end{array}$ & $\begin{array}{l}\text { Single stage revision } \\
\qquad \mathrm{N}=5\end{array}$ & $\begin{array}{c}\text { Two stage revision } \\
\mathrm{N}=11\end{array}$ & $\begin{array}{c}\text { Statistical difference (One } \\
\text { way ANOVA*, } \\
\text { Chi-squared test**) }\end{array}$ \\
\hline Age mean (years) & 72 & 54 & 64 & $0.08^{*}$ \\
\hline Sex (M:F ratio) & $4: 2$ & $2: 3$ & $3: 8$ & $0.29 * *$ \\
\hline At risk $\mathrm{PMH}$ & $33.3 \%$ & $60 \%$ & $45.5 \%$ & $0.68 * *$ \\
\hline Joint type (Hip:Knee ratio) & $1: 5$ & $2: 3$ & $2: 9$ & $0.58 * *$ \\
\hline Acute:chronic PJI ratio & $5: 1$ & $1: 4$ & $1: 10$ & $0.006 * *$ \\
\hline $\begin{array}{l}\text { Time between presentation and } 1^{\text {st }} \\
\text { surgery (days) }\end{array}$ & 55.7 & 100 & 536 & $0.01 *$ \\
\hline Time between $1^{\text {st }}$ and $2^{\text {nd }}$ surgery (days) & 0 & 0 & $123(\mathrm{~N}=8)$ & $0.0002 *$ \\
\hline
\end{tabular}

Significant values shown in BOLD. 
Table 2. - Diagnostic accuracy of ADLF testing at $1^{\text {st }}$ and $2^{\text {nd }}$ stage sampling

\begin{tabular}{|l|c|c|c|c|}
\hline & Sensitivity & Specificity & PPV & NPV \\
\hline $\begin{array}{l}\text { DAIR } \\
\mathbf{1}^{\text {st }} \text { sample }\end{array}$ & $66.6 \%$ & $0 \%$ & $100 \%$ & $0 \%$ \\
\hline $\begin{array}{l}\text { Single stage } \\
\mathbf{1}^{\text {st }} \text { sample }\end{array}$ & $66.6 \%$ & $100 \%$ & $100 \%$ & $66.6 \%$ \\
\hline $\begin{array}{l}\text { Two stage } \\
\mathbf{1}^{\text {st }} \text { sample }\end{array}$ & $20 \%$ & $0 \%$ & $33.3 \%$ & $0 \%$ \\
\hline $\begin{array}{l}\text { Total } \\
\mathbf{1}^{\text {st }} \mathbf{s a m p l e}\end{array}$ & $50 \%$ & $50 \%$ & $77.8 \%$ & $22.2 \%$ \\
\hline $\begin{array}{l}\text { DAIR } \\
\mathbf{2}^{\text {nd }} \text { sample }\end{array}$ & $50 \%$ & $100 \%$ & $100 \%$ & $75 \%$ \\
\hline $\begin{array}{l}\text { Single stage } \\
\mathbf{2}^{\text {nd }} \text { sample }\end{array}$ & $0 \%$ & $100 \%$ & $0 \%$ & $100 \%$ \\
\hline $\begin{array}{l}\text { Two stage } \\
\mathbf{2}^{\text {nd }} \text { sample }\end{array}$ & $0 \%$ & $100 \%$ & $0 \%$ & $37.5 \%$ \\
\hline $\begin{array}{l}\text { Total } \\
\mathbf{2}^{\text {nd }} \text { sample }\end{array}$ & $14.3 \%$ & $100 \%$ & $100 \%$ & $62.5 \%$ \\
\hline
\end{tabular}

For the ADLF testing at the $2^{\text {nd }}$ stage sampling $5.9 \%(\mathrm{~N}=1)$ were true positives and $58.8 \%(\mathrm{~N}=10)$ were true negatives, there were no false positive and $35.5 \%(\mathrm{~N}=6)$ were false negative. Using ADLF testing to predict eradication of infection following surgical intervention had 100\% specificity and PPV, $14.3 \%$ sensitivity and $62.5 \%$ NPV.

Four patients had not had ADLF testing at the first sampling i.e. intra-operatively prior to surgical intervention, so these patients were excluded from the subsequent analysis.

For the ADLF testing at the $1^{\text {st }}$ stage sampling $38.9 \%$ of samples $(\mathrm{N}=7)$ had a true positive result, $11.1 \%(\mathrm{~N}=2)$ were true negative compared to $11.1 \%(\mathrm{~N}=2)$ false positives and $38.9 \%(\mathrm{~N}=7)$ false negatives. ADLF testing of intra-operative samples used to predict PJI had 50\% sensitivity and specificity, $77.8 \%$ PPV and 22.2\% NPV.

\section{DISCUSSION}

This prospective analysis supports the hypothesis that alpha defensin in-theatre lateral flow test, is most useful in terms of specificity, PPV and NPV when utilised at the end of the surgical debridement to confirm absence of residual infection.
There are key limitations of this work. Numbers in this cohort were small and the overall cohort number was reduced further for some analyses as four of the two stage revision patients had not had ADLF testing at their first stage as they were recruited after this point and five patients who had had first stage testing did not proceed to a second stage revision. However, the investigation was run as a pilot study with the aim of leading to a larger cohort study if the initial results indicated the ADLF could have a role in PJI revision arthroplasty surgery. The current study also experienced difficulty obtaining the second fluid samples following the debridement and washout of the DAIR and single stage revision procedures, due to small volumes of fluid being present following the initial debridement. Data from the National Joint Registry recorded from 2004 to 2014 on revision procedures performed as a consequence of PJI has shown that the percentage of patients having DAIR or single stage revision surgeries has increased from $0.4 \%$ to $7.6 \%$ and from $18.1 \%$ to $29.7 \%$ respectively (17) so investigating the use of ADLF in these procedures was felt to be increasingly applicable to current orthopaedic practice.

The current study reported ADLF at first sampling to have a sensitivity lower than that previously reported by Kasparek et al.(18) however, these workers used the Musculoskeletal Infection Society (MSIS) criteria (4) as their standard for comparison which could partly explain the discrepancy between the reported sensitivity rates in the current study. The MSIS criteria (19) was not used as the gold standard in the current study as the majority of patients did not have all investigations completed to use this criteria. Concerns about the practicality of applying the MSIS criteria to routine clinical use, including cost and time implications, have previously been raised (20).

Renz et al. (21) reported a lower rate of sensitivity compared to previous reports $(11,18)$. This was attributed to the inclusion of chronic PJI cases (21). A further reason for the inferior sensitivity rates of the current study could be the use of intra-operative samples for ADLF testing. Balato et al. (22) reported superior sensitivity rates in cases of chronic PJI of the knee however, they used pre-operative aspirate 
samples as well as excluding patients that had received antibiotics during the preceding two weeks and they used the MSIS criteria as a standard for comparison, rather than the final culture results.

The use of ADLF in revision surgery for PJI has been previously investigated $(11,23)$ and high rates of specificity have been reported consistent with the findings of the current study. The review of second stage sampling performed in the current study reported inferior rates of sensitivity compared to Sigmund et al. (23) and Berger et al. (11) This could partly be explained by the inclusion of patients with cement spacers and testing of samples taken following the implantation of spacers had a sensitivity of $0 \%$, however this is not consistent with the previously reported findings by Shahi et al. (14) that alpha defensin levels are not effected by antibiotic administration. The poor sensitivity reported by the current study concurs with the recent work from Scholten et al. (24) which assessed the diagnostic accuracy of ADLF testing when used intra-operatively rather than with a pre-operative aspirate.

In this work ADLF used at the end of the surgical treatment for PJI had high specificity and PPV. Given that at this stage the sole clinical focus is on exclusion of residual infection following presumptive eradication then high indices of reliability support the role of this binary test in the decision making process prior to re-implantation. However, the current study did have incidences of culture positive second sampling, including five two stage revision patients having culture positive samples, but ADLF negative tests, at the reimplantation stage. It has been reported that positive cultures may still be present in up to $20 \%$ of hip and knee PJI cases undergoing two stage revision procedure at the second stage (25). Furthermore, $63 \%$ of them may develop infection again over time (25). Therefore, a test with high rates of both sensitivity and specificity may be required at the second stage rather than just a test of exclusion.

\section{CONCLUSION}

This current work found a high rate of specificity and PPV for the use of ADLF at the second stage of surgical treatment for PJI indicating that it may have potential use as a test of exclusion.

\section{REFERENCES}

1. Munemoto M, Inagaki Y, Tanaka Y, Grammatopoulos G, Athanasou NA. Quantification of neutrophil polymorphs in infected and noninfected second-stage revision hip arthroplasties. Hip Int 2016 ; 26(4) : 327-30.

2. Beaule PE, Shea B, Abedlbary H, Ahmadzai N, Skidmore B, Mallick R, et al. A protocol for a systematic review of the diagnostic accuracy of blood markers, synovial fluid, and tissue testing in periprosthetic joint infections (PJI). Syst Reviews 2015 ; 4(1) : 148.

3. George D, Gant V, Haddad F. The management of periprosthetic infections in the future : a review of new forms of treatment. Bone Joint J 2015 ; 97(9) : 1162-9.

4. Gehrke T, Parvizi J. Proceedings of the international consensus meeting on periprosthetic joint infection. $J$ Arthroplasty $2014 ; 29(2): 4$.

5. Drago L, Toscano M, Tacchini L, Banfi G. $\alpha$-Defensin point-of-care test for diagnosis of prosthetic joint infections : neglected role of laboratory and clinical pathologists. Clin Chem Lab Med 2017 ; 56(1) : 19-24.

6. Parvizi J, Adeli B, Zmistowski B, Restrepo C, Greenwald AS. Management of Periprosthetic Joint Infection: The Current Knowledge AAOS Exhibit Selection. J Bone Joint Surg 2012 ; 94(14) : e104.

7. Osmon DR. Microbiology and antimicrobial challenges of prosthetic joint infection. J Am Acd Orthop Surg 2017 ; 25 : S17-S9.

8. Squire MW, Valle CJD, Parvizi J. Preoperative diagnosis of periprosthetic joint infection : role of aspiration. Am J Roent 2011 ; 196(4) : 875-9.

9. Ganz T, Selsted ME, Szklarek D, Harwig S, Daher K, Bainton DF, et al. Defensins. Natural peptide antibiotics of human neutrophils. J Clin Invest 1985 ; 76(4) : 1427-35.

10. Deirmengian C, Kardos K, Kilmartin P, Gulati S, Citrano P, Booth RE. The alpha-defensin test for periprosthetic joint infection responds to a wide spectrum of organisms. Clin Orthop 2015 ; 473(7) : 2229-35.

11. Berger P, Van Cauter M, Driesen R, Neyt J, Cornu O, Bellemans J. Diagnosis of prosthetic joint infection with alpha-defensin using a lateral flow device : a multicentre study. Bone Joint J 2017 ; 99(9) : 1176-82.

12. Suda AJ, Tinelli M, Beisemann ND, Weil Y, Khoury A, Bischel OE. Diagnosis of periprosthetic joint infection using alpha-defensin test or multiplex-PCR : ideal diagnostic test still not found. Int Orthop 2017 ; 41(7) : 130713.

13. Newman JM, George J, Klika AK, Hatem SF, Barsoum WK, North WT, et al. What is the diagnostic accuracy of aspirations performed on hips with antibiotic cement spacers? Clin Orthop 2017 ; 475(1) : 204-11. 
14. Shahi A, Parvizi J, Kazarian GS, Higuera C, Frangiamore S, Bingham $\mathbf{J}$, et al. The alpha-defensin test for periprosthetic joint infections is not affected by prior antibiotic administration. Clin Orthop 2016 ; 474(7) : 1610-5.

15. Diagnostics C. Synovasure (R) Alpha Defensin Lateral Flow Test Kit. In: Biomet Z, editor. V3.3 ed: CD Diagnostics.

16. Bozic KJ, Lau E, Kurtz S, Ong K, Berry DJ. Patientrelated risk factors for postoperative mortality and periprosthetic joint infection in medicare patients undergoing TKA. Clin Orthop 2012 ; 470(1) : 130-7.

17. Lenguerrand E, Whitehouse M, Beswick A, Jones S, Blom A. Revision for prosthetic joint infection following hip arthroplasty. Bone 2017 ; 3 : R1.

18. Kasparek MF, Kasparek M, Boettner F, Faschingbauer M, Hahne J, Dominkus M. Intraoperative diagnosis of periprosthetic joint infection using a novel alpha-defensin lateral flow assay. J Arthroplasty 2016 ; 31(12) : 2871-4.

19. Parvizi J, Zmistowski B, Berbari EF, Bauer TW, Springer BD, Della Valle CJ, et al. New definition for periprosthetic joint infection : from the Workgroup of the Musculoskeletal Infection Society. Clin Orthop 2011; 469(11) : 2992.

20. Wang C, Li R, Wang Q, Duan J, Wang C. Leukocyte esterase as a biomarker in the diagnosis of periprosthetic joint infection. Med Sci Monitor $2017 ; 23: 353$.
21. Renz N, Yermak K, Perka C, Trampuz A. Alpha Defensin Lateral Flow Test for Diagnosis of Periprosthetic Joint Infection : Not a Screening but a Confirmatory Test. J Bone Joint Surg 2018 ; 100(9) : 742-50.

22. Balato G, Franceschini V, Ascione T, Lamberti A, D'Amato M, Ensini A, et al. High performance of $\alpha$-defensin lateral flow assay (Synovasure) in the diagnosis of chronic knee prosthetic infections. Knee Surg Sports Tramatol Arthrosc 2018 ; 26(6) : 1717-22.

23. Sigmund I, Holinka J, Gamper J, Staats K, Böhler C, Kubista B, et al. Qualitative $\alpha$-defensin test (Synovasure) for the diagnosis of periprosthetic infection in revision total joint arthroplasty. Bone Joint J 2017 ; 99(1) : 66-72.

24. Scholten R, Visser J, Van Susante JL, Van Loon CJ. Low sensitivity of a-defensin (Synovasure) test for intraoperative exclusion of prosthetic joint infection. Acta Orthop 2018 ; 89(3) : 357-9.

25. Sorlí L, Puig L, Torres-Claramunt R, González A, Alier A, Knobel H, et al. The relationship between microbiology results in the second of a two-stage exchange procedure using cement spacers and the outcome after revision total joint replacement for infection : the use of sonication to aid bacteriological analysis. J Bone Joint Surg Br 2012 ; 94(2) : 249-53. 